\title{
An improved weighted essentially non-oscillatory scheme with modified smoothness indicator $\tau$ and adaptive index $p$
}

Xin Li, Chang-Ping Yu, Li Li \& Xin-Liang Li

To cite this article: Xin Li, Chang-Ping Yu, Li Li \& Xin-Liang Li (2020): An improved weighted essentially non-oscillatory scheme with modified smoothness indicator $\tau$ and adaptive index $p$, International Journal of Computational Fluid Dynamics, DOI: 10.1080/10618562.2020.1754403

To link to this article: https://doi.org/10.1080/10618562.2020.1754403

Published online: 23 Apr 2020.

Submit your article to this journal ऍ

Q View related articles $\square$

View Crossmark data ¿ 


\title{
An improved weighted essentially non-oscillatory scheme with modified smoothness indicator $\tau$ and adaptive index $p$
}

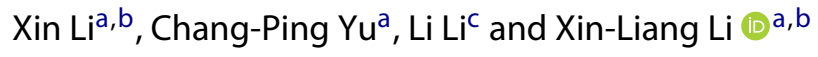 \\ aLHD, Institute of Mechanics, Chinese Academy of Sciences, Beijing, People's Republic of China; b School of Engineering Science, University of \\ Chinese Academy of Sciences, Beijing, People's Republic of China; ' Laboratory of Computational Physics, Institute of Applied Physics and \\ Computational Mathematics, Beijing, People's Republic of China
}

\section{ABSTRACT}

The resolution and robustness properties of a numerical scheme are two mutually restricted aspects for the detailed simulation of complex flows. In the present paper, an improved seventh-order finite difference weighted essentially non-oscillatory scheme (WENO-PR) is developed by distributing larger weights to the less smooth stencils. To meet the optimization requirements, the high-order smoothness indicator $\tau$ is modified by some constraints and a real-number index factor $p$ is designed under the control of a wavenumber identifier. The results show that the modifications in the proposed scheme improve self-adaptation features in the smooth and discontinuous regions, which greatly balance the numerical resolution and robustness. Compared with the existing schemes such as WENO-Z and WENO-JS, the WENO-PR is more flexible, accurate and robust. Several one- and two-dimensional numerical tests are performed to confirm the high-resolution capability and the non-oscillatory property of the proposed scheme.

\section{ARTICLE HISTORY}

Received 5 September 2019

Accepted 15 January 2020

\section{KEYWORDS}

WENO scheme; high resolution; shock-capturing; smoothness indicator; adaptive optimisation

\section{Introduction}

Currently, many studies are focussing on detailed simulations of compressible complex flows, such as direct numerical simulation (DNS) and large eddy simulation (LES). For the DNS and LES of compressible turbulent flows, numerical schemes should possess non-oscillatory properties to capture shock waves that characterise the flows. Additionally, these schemes should provide an accurate representation of the varying ranges of flow details, including high-frequency small-scale turbulent fluctuations and multi-scaled turbulent vortices. Significantly low dissipation may lead to numerical oscillation and computation overflow. Hence, researchers need to resolve the contradiction between shock-capturing capabilities and low dissipation.

Several numerical methods have been developed to resolve this contradiction. Harten (1983) and Shu (1987) proposed a second-order accurate total variation diminishing (TVD) scheme and an improved total variation bounded (TVB) scheme, respectively.
Subsequently, a successful high-order method, essentially non-oscillatory schemes (ENO), was introduced (Harten et al. 1986), which was used to resolve reduced-order problems (Harten 1989; Harten et al. 1987). The ENO scheme maintains the higher order by cancelling the monotonicity-preserving limit of the TVD, and the substencils with high-order interpolation are adaptively selected to reconstruct numerical flux to avoid spurious oscillation (Shu and Osher 1988, 1989). However, some effective information is wasted when selecting the smoothest stencil and discarding others. To overcome this drawback, Liu, Osher, and Chan (1994) proposed the first weighted ENO scheme (WENO) by introducing a weight function to combine the information on all candidate substencils. Furthermore, a finite difference WENO scheme (WENO-JS) was constructed (Jiang and Shu 1996; Balsara and Shu 2000), in which a practical smoothness indicator was designed to improve computational efficiency, but the WENO-JS scheme was still too dissipative for the detailed simulations of high-frequency fluctuations

CONTACT Xin-Liang Li @ lixl@imech.ac.cn 
in flow fields (Liu et al. 2015). Henrick, Aslam, and Powers (2005) clearly pointed out that the WENOJS scheme failed to recover the maximum order at critical points. In their study, a correcting mapping function that approximating the WENO-JS scheme to an original linear scheme was devised, leading a resulting mapped WENO scheme called WENO-M recovered the optimal order of accuracy at critical points. Subsequently, Martín et al. (2006) proposed the WENO-SYMBO and the WENO-SYMBOO schemes which rely on symmetrical stencils and discriminant functions. While these schemes have been successfully applied, they possess reduced robustness (Wu and Martín 2007). In an attempt to perform high-fidelity simulation of turbulence, a meaningful high-order targeted ENO scheme (TENO) was proposed by assembling the low-order substencils with incrementally increasing width ( $\mathrm{Fu}, \mathrm{Hu}$, and Adams 2016). Thereafter, the TENO scheme was extended to the eighthorder TENO scheme (Fu, Hu, and Adams 2017), the dispersion and dissipation properties of which were optimised independently in a unified framework. Furthermore, the TENO scheme was improved by an adaptation parameter $C_{T}$ for effectively minimising numerical dissipation and maintaining robustness for shock-capturing ( $\mathrm{Fu}, \mathrm{Hu}$, and Adams 2018). In addition, various improved versions of the WENO schemes have been launched (Ha et al. 2013; Kim, Ha, and Yoon 2016; Gande, Rathod, and Rathan 2017; Li et al. 2017; Rathan and Raju 2018) and have been widely used in different simulations (Zhou, He, and Shen 2012; Tong et al. 2017; Kitamura and Nonomura 2017).

An effective scheme, named as WENO-Z, was developed by Borges et al. (2008). The study succeeded in modifying the low-order smoothness indicator by designing a coefficient of higher-order smoothness indicator $\tau$ to examine overall accuracy. Under the action of $\tau$, the WENO-Z scheme achieves the desired order of convergence at critical points. The index factor $p$ is dependent on the accuracy and the dissipation of the numerical scheme, that is, a smaller $p$ leads to less dissipation, lower robustness and vice-versa. For $p=1$, the WENO-Z is not sufficiently robust for practical cases and reduces one order at the critical points in smooth regions. For $p=2$, the scheme recovers maximum order with higher numerical dissipation. However, although the index $p$ attains larger values in $[1, r-1]$, the robustness of WENO-Z is weaker than the classical WENO-JS. Castro, Costa, and Don (2011) extended this scheme to the $2 r-1$ order and obtained a general formula, wherein the smoothness indicators $\tau$ and $p$ have been considered together. It is found that the discontinuities can be identified by using a more effective $\tau$, and the scheme with $p=r-1$ has no visible oscillation owing to the large dissipation. Hence, these modifications essentially increase the weights of the discontinuous stencils. Recently, a sixth-order WENO-CU scheme with a sixth-order $\tau_{6}$ and a fifthorder WENO- $\eta$ scheme with $\tau$ based on Lagrange interpolation were proposed by $\mathrm{Hu}$, Wang, and Adams (2010) and Fan et al. (2014), respectively. Subsequently, a WENO-AMM scheme was developed by getting some constraints on the nonlinear weights to guarantee maximal order accuracy (Aràndiga, Martí, and Mulet 2014). Later, a problem-independent nonlinear switch was investigated (Arshed and Khan 2019) and a new global smoothness indicator was obtained by applying the Taylor expansion to the local smoothness indicator ( $\mathrm{Li}, \mathrm{Li}$, and $\mathrm{Ge} 2019$ ). For approximating the derivative of a smooth function with high order critical points, two improved WENO-D and WENO-A schemes employing sensitivity parameters were developed (Wang, Wang, and Don 2019). These schemes are based on the WENO-Z scheme and achieve an improved balance between resolution and robustness.

Based on the discussion mentioned above, WENO$\mathrm{Z}$ depends on the expression of the $\tau$ and the selection of the integer index $p$, which enlightens this study to explore the abilities of $\tau$ and $p$ to reassign the weights for further optimisation. The smoothness indicator $\tau$ is initially modified for less numerical dissipation, following which the index $p$ is extended to the real number domain using a wavenumber identifier. The improved $\tau$ and adaptive $p$ are determined via numerical characteristic analyses.

This remainder of this paper is organised as follows. In Section 2 and Section 3, reviews of the WENOJS and WENO-Z schemes are provided. The proposed WENO-PR scheme is described in Section 4, with detailed discussions on the structuring of the new scheme. In Section 5, the numerical characteristics of the WENO-PR are discussed. Section 6 presents the assessment of the scheme and its validation based on the benchmark cases involving discontinuities and multi scales; more accurate numerical solutions and better no-oscillatory properties than those of the WENO-JS and WENO-Z schemes are achieved by 
the proposed scheme. Finally, we conclude our results in Section 7.

\section{WENO-JS scheme}

In this section, a brief description of the seventhorder WENO-JS scheme employing four 4-points substencils is provided, as shown in Figure $1 . H_{j+1 / 2}^{(k)}$ is computed by the fourth-order polynomial interpolation for each stencil, and all of them are combined with weight functions for the overall seventh-order accuracy in the smooth region.

For simplicity, the one-dimensional scalar hyperbolic conservation law can be expressed by:

$$
\frac{\partial u}{\partial t}+\frac{\partial f(u)}{\partial x}=0, f(u)=a u, a>0
$$

On uniform grids, it can be written as a semi-discrete conservative finite difference scheme in the form of the WENO scheme:

$$
\left.\frac{\partial u}{\partial t}\right|_{j}=\frac{f_{j+1 / 2}^{W E N O}-f_{j-1 / 2}^{W E N O}}{\Delta x}
$$

where $f_{j+1 / 2}^{W E N O}$ is the numerical flux at the semi-point $j+1 / 2$. For the seventh-order WENO-JS, the numerical flux is computed as:

$$
\begin{aligned}
f_{j+1 / 2}^{W E N O-J S} & =\sum_{k=0}^{3} \omega_{k} H_{j+1 / 2}^{(k)}, \\
H_{j+1 / 2}^{(k)} & =\sum_{k=0}^{3} \alpha_{l}^{(k)} f_{j-k+l},
\end{aligned}
$$

where $H_{j+1 / 2}^{(k)}$ is the fourth-order polynomial interpolation for the $k^{\text {th }}$ stencil, $k=0,1,2,3$. The weight $\omega_{k}$ is

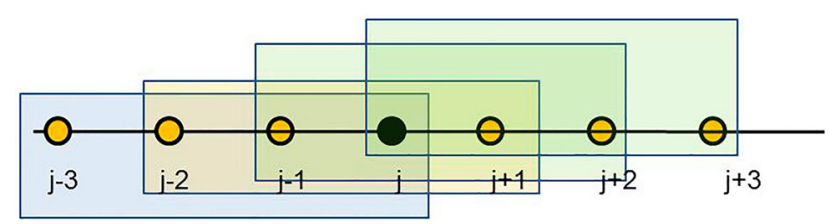

Figure 1. Computational grids and the four 4-points stencils of the seventh-order WENO-JS scheme. defined by:

$$
\begin{aligned}
\omega_{k} & =\frac{\alpha_{k}}{\sum_{k=0}^{3} \alpha_{l}}, \\
\alpha_{k} & =\frac{C_{k}}{\left(\beta_{k}+\varepsilon\right)^{p}}, \\
C_{0} & =\frac{1}{35}, C_{1}=\frac{12}{35}, C_{2}=\frac{18}{35}, C_{3}=\frac{4}{35}
\end{aligned}
$$

where $C_{k}$ is an ideal weight. The constant $\epsilon$ has a small value of $10^{-7}$ to avoid division by zero. The parameter $p$ ensures adaptation sensitivity to discontinuities and $p=2$ is usually chosen to ensure numerical stability. The smooth degree of the flow field is measured using the low-order smoothness indicator $\beta_{k}$ given by:

$$
\beta_{k}=\sum_{k=0}^{3} \int_{x_{j-1 / 2}}^{x_{j+1 / 2}} \Delta x^{2 l-1}\left(\frac{\partial^{l}}{\partial x^{l}} q_{k}(x)\right)^{2} \mathrm{~d} x
$$

where the function $q_{k}(x)$ is interpolated by the corresponding stencil.

In the smooth regions, because $\omega_{k}$ is a good approximation to the ideal weight resulting from the small and similar value of $\beta_{k}$, WENO-JS tends to the seventh-order upwind spatial discretization scheme. $\beta_{k}$ substantially increases when discontinuities occur, so the weights in the continuous regions become significant in all stencils. In WENO-JS, the weights in the continuous regions are assigned larger values than others, thereby enhancing the numerical dissipation and reducing second-order accuracy near discontinuities.

\section{WENO-Z scheme}

Based on the WENO-JS, the WENO-Z scheme is developed to recover high order near critical points. The numerical flux of the seventh-order WENO-Z is:

$$
f_{j+1 / 2}^{W E N O-Z}=\sum_{k=0}^{3} \omega_{k}^{Z} H_{j+1 / 2}^{(k)}
$$

where $H_{j+1 / 2}^{(k)}$ is the numerical flux of each candidate stencil. The nonlinear weight $\omega_{k}^{Z}$ of the $k^{\text {th }}$ substencil 
$(k=0,1,2,3)$ is given by:

$$
\begin{aligned}
\omega_{k}^{Z} & =\frac{\alpha_{k}^{Z}}{\sum_{k=0}^{3} \alpha_{l}^{Z}}, \\
\alpha_{k}^{Z} & =\frac{C_{k}}{\beta_{k}^{Z}}=C_{k}\left[1+\left(\frac{\tau_{7}}{\varepsilon+\beta_{k}}\right)^{p}\right], \\
\beta_{k}^{Z} & =\left(\beta_{k}+\varepsilon\right) /\left(\beta_{k}+\tau_{7}+\varepsilon\right), \\
\tau_{7} & =\max \left(\left|\beta_{0}+3 \beta_{1}-3 \beta_{2}-\beta_{3}\right|, \varepsilon\right),
\end{aligned}
$$

The increasing $p$ leads to larger numerical dissipation and more robustness, while it is often considered as 1 and 2 in Equation (11). The scheme is dissipative to prevent spurious oscillation when $p=r-1$ and it has $r=4$ for the seventh-order WENO-Z. The terms $\beta_{k}$ and $H_{j+1 / 2}^{(k)}$ are the same as those in the WENO-JS. Following the features of the high-order smoothness indicator $\tau_{7}$ obtained by Don and Borges (2013), we have:

- In the smooth stencils, $\tau_{7}=\mathrm{O}\left(\Delta x^{7}\right)$;

- In the stencils with discontinuities, if the $k^{\text {th }}$ substencil is relatively smooth, $\beta_{k}$ is less than $\tau_{7}$;

- $\tau_{7} \leq \max _{k} \beta_{k}$.

The nonlinear parameter $\alpha_{k}^{Z}$ is slightly modified by replacing $\beta_{k}$ with $\beta_{k}^{Z}$ to obtain higher robustness (Wang and Wu 2015), and the new $\alpha_{k}^{Z}$ is defined by:

$$
\alpha_{k}^{Z}=C_{k}\left[1+\left(\frac{\tau_{7}}{\varepsilon+\beta_{k}^{Z}}\right)^{p}\right],
$$

where $\beta_{k}^{Z}$ is given by Equation (12).

\section{Proposed WENO-PR scheme}

The procedure for the construction of the WENO-PR is presented in this section. The high order smoothness indicator $\tau_{7}$ is modified, and the expression of $p$ based on the local smoothness is designed for adaptive dissipation.

\subsection{Modification of $\tau_{7}$}

As demonstrated in Section III, if $\beta_{k}$ is less than $\tau_{7}$ at the discontinuities, $\alpha_{k}^{Z}$ increases and the corresponding $\omega_{k}^{Z}$ becomes a dominant value. Therefore, the differences in each $\omega_{k}^{Z}$ can be reduced by limiting the maximum difference between $\beta_{k}$ and $\tau_{7}$. The complete scheme is approximately linear when the $\omega_{k}^{Z}$ increases at discontinuities. For this purpose, $\tau_{7}$ is replaced by $Z_{p r}$ atan $\left(\tau_{7}\right)$ with the maximum absolute value limited by $Z_{p r} \bullet \pi / 2$, where atan represents the function of arc tangent and $Z_{p r}$ is an artificial parameter called limit coefficient which indicates the similarity between $\beta_{k}$ and $\tau_{7}$. The modification of atan decreases the differences between $\tau_{7}$ and $\omega_{k}^{Z}$ at discontinuities, resulting in the new nonlinear parameter as:

$$
\alpha_{k}^{Z}=C_{k}\left[1+\left(\frac{Z_{p r} \operatorname{atan}\left(\tau_{7}\right)}{\beta_{k}^{Z}+\varepsilon}\right)^{p}\right],
$$

where $\beta_{k}^{Z}$ is defined as shown in Equation (12).

To estimate the influence of atan and $Z_{p r}$, the nonlinear spectral analysis proposed by Pirozzoli (2002) is adopted. In Figure 2, the solid black lines represent ideal dispersion and dissipation, respectively. The dashed lines depict the ranges of the upper-lower limit of the results with these selected parameters. It is found that the dispersion and dissipation properties are optimised with the modification of atan and depend on $Z_{p r} . K_{i}$ and $K_{r}$ are approximately the ideal situations with a decreasing $Z_{p r}$, as indicated by the arrows. Although atan leads to few changes, it is necessary to add it first. A finite value is given as the limitation of atan to avoid a significantly large value of $\tau_{7}$, which is the foundation of the control of $Z_{p r}$. However, owing to a significantly small $Z_{p r}$ may lead to high numerical oscillation, a compromise value, $Z_{p r}=0.3 /(2 \pi)$, is chosen to balance the dispersion and dissipation, whose results are at the centre of the two dashed black lines, as shown in Figure 2. Here, $Z_{p r}$ is an adjustable parameter, whose magnitude can be measured by treating $\pi$ as a unit.

\subsection{Adaptive $p$}

From linearisation owing to modification of $\tau_{7}$, certain numerical oscillations may occur in the high-wavenumber regions. These oscillations can be removed by modifying the index $p$ that plays an important role in WENO adaptation sensitivity. Previously, $p$ is chosen as an integer such as $p=1$ and 2. To ensure a small amount of dissipation and the stability in low- and high-wavenumber regions, respectively, an adaptive $p$ will be considered.

The numerical oscillation of the WENO scheme disappears when $p=r-1$. For $r=4$ adopted in the 


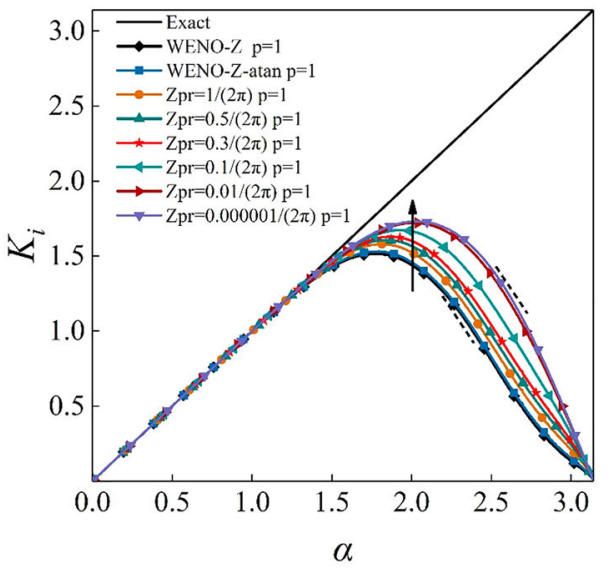

(a)

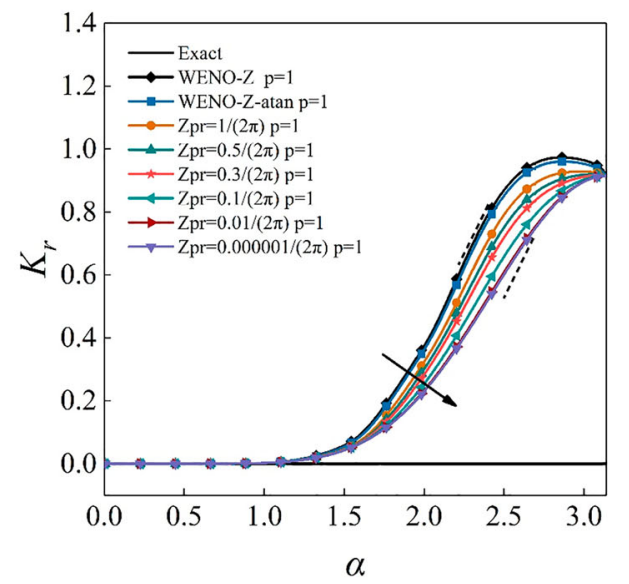

(b)

Figure 2. Numerical characteristics with different $\tau$ 7. (a) Dispersion. (b) Dissipation.

seventh-order WENO scheme, $p=3$ is chosen as the upper limit of the adaptive $p$. In order to reduce the dissipation error and ensure better resolution property than the WENO-Z scheme, a compromise minimum value $p=0.5$ between 0 and 1 is chosen. Thus, an adaptive $p$ whose range is in the interval $[0.5,3]$ is further designed as a linear function of $\theta_{j+1 / 2}$ and can be expressed as:

$$
p=0.5+2.5 \theta_{j+1 / 2}
$$

where $\theta_{j+1 / 2}$ is a numerical wavenumber identifier ( $\mathrm{Li}$ et al. 2016) defined by:

$$
\begin{aligned}
\theta_{j+1 / 2} & =\frac{\left|S_{1}^{r}-S_{2}^{r}\right|^{s}}{\left|S_{1}^{r}+S_{2}^{r}\right|^{s}} \\
S_{1} & =\left|u_{j+1}+u_{j-1}\right| \\
S_{1} & =\left|u_{j+2}+u_{j+1}-u_{j}-u_{j-1}\right| / 4
\end{aligned}
$$

As shown in Figure 3, the identifier $\theta_{j+1 / 2}$ with $r=4$ and $s=1$ recommended by Li monotonously increases with the increasing wavenumber $\alpha \equiv k \Delta x$. It can be seen that $\theta_{j+1 / 2}$ varies in the range from 0 to 1 in the smooth region (low-wavenumber region) and the discontinuous region (high-wavenumber region). The location of $\theta=0.98$ is depicted by a short dash line indicating a high-wavenumber region.

Compared with the constants $p=0.5$ and 3 , the dispersion and dissipation properties of the scheme with the adaptive $p$ are shown in Figure 4. It is observed that the dispersion and dissipation with the adaptive $p$ are similar to that for $p=3$, although they are not completely coincident. Furthermore, the adaptive $p$ ensures that the dissipation is non-negative in

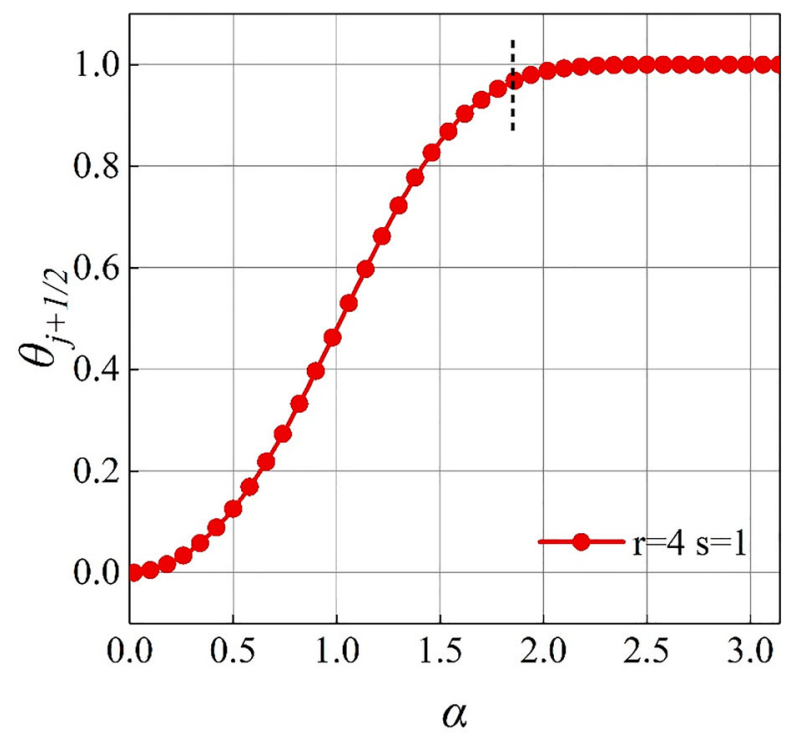

Figure 3. Values of $\theta_{j+1 / 2}$ as a function of the wavenumber $\alpha$.

the high-wavenumber region and has similar characteristics as those for $p=0.5$ in the low-wavenumber region. The intersected location is depicted by a short horizontal dash line, which is consistent with Figure 3.

\subsection{Expression of WENO-PR}

Here, we summarise the final expressions of the WENO-PR scheme which can be written into the uniform framework as:

$$
f_{j+1 / 2}^{W E N O-P R}=\sum_{k=0}^{3} \omega_{k}^{Z} H_{j+1 / 2}^{(k)}
$$




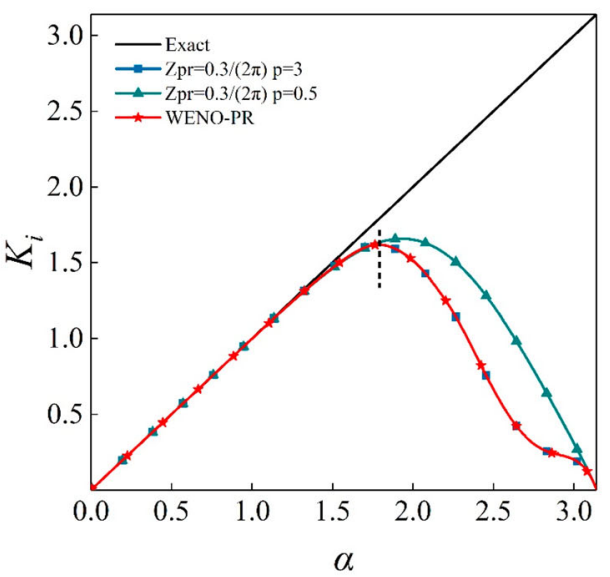

(a)

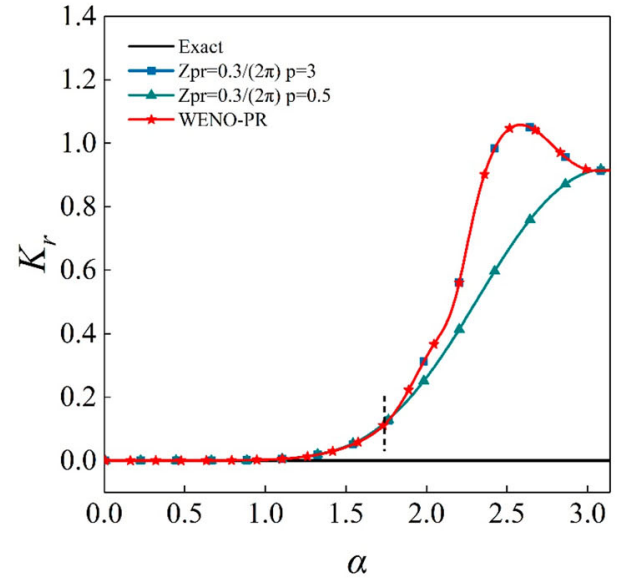

(b)

Figure 4. Numerical characteristics with the adaptive $p$. (a) Dispersion. (b) Dissipation.

where the nonlinear weight is:

$$
\omega_{k}^{Z}=\frac{\alpha_{k}^{Z}}{\sum_{k=0}^{3} \alpha_{l}^{Z}},
$$

Other related functions are:

$$
\begin{aligned}
\alpha_{k}^{Z} & =C_{k}\left[1+\left(\frac{Z_{p r} \operatorname{atan}\left(\tau_{7}\right)}{\beta_{k}^{Z}+\varepsilon}\right)^{p}\right], \\
\beta_{k}^{Z} & =\left(\beta_{k}+\varepsilon\right) /\left(\beta_{k}+\tau_{7}+\varepsilon\right), \\
\tau_{7} & =\max \left(\left|\beta_{0}+3 \beta_{1}-3 \beta_{2}-\beta_{3}\right|, \varepsilon\right),
\end{aligned}
$$

where $p$ is defined as:

$$
\begin{aligned}
p & =0.5+2.5 \theta_{j+1 / 2}, \\
\theta_{j+1 / 2} & =\frac{\left|S_{1}^{4}-S_{2}^{4}\right|}{\left|S_{1}^{4}+S_{2}^{4}\right|} \\
S_{1} & =\left|u_{j+1}+u_{j-1}\right|, \\
S_{1} & =\left|u_{j+2}+u_{j+1}-u_{j}-u_{j-1}\right| / 4,
\end{aligned}
$$

The terms $\omega_{k}^{Z}, C_{k}, \beta_{k}$, and $H_{j+1 / 2}^{(k)}$ have the same forms and meanings as those in WENO-Z. In WENO$\mathrm{PR}$, only the limit coefficient $Z_{p r}$ which is recommended as $Z_{p r}=0.3 /(2 \pi)$ is an adjustable parameter and the other parameters are fixed.

\section{Analysis of the numerical characteristics}

\subsection{Nonlinear spectral analysis}

Figure 5 provides a spectral study of the WENO-PR scheme, compared with the WENO-JS and WENO$\mathrm{Z}$ schemes with different $p$. It is observed that the results from WENO-PR coincide with the ideal solutions in the lower-wavenumber region, thereby indicating better dissipation and dispersion properties. In the medium-wavenumber region for $1.5 \leq \alpha \leq 2$, the results of WENO-PR are more similar to the ideal results by comparison with the other two schemes. Additionally, in the higher-wavenumber region, the results of WENO-PR are between WENO-JS and WENO-Z, indicating that a certain amount of dissipation is introduced to ensure the stability of the scheme. These characteristics are considered in practical simulations.

\subsection{Weights analysis}

To further illustrate the changes after applying the adaptive $p$, the non-linear weights of the substencils on the discontinuities are analysed. Figure 6 presents the first-order derivatives of the discontinuous function in the first-time step.

$$
\begin{aligned}
u(x, 0) & =f(x) \\
& =\left\{\begin{array}{l}
-\sin (\pi x)-\frac{1}{2} x^{3}, \quad-1 \leq x \leq 0 \\
-\sin (\pi x)-\frac{1}{2} x^{3}+1, \quad 0 \leq x \leq 1
\end{array}\right.
\end{aligned}
$$

It is observed that when a substencil encounters a discontinuity, the corresponding weight deviates from the ideal situation and becomes smaller. As $p$ increases, the weights are fixed at zero from $x=-0.01$ to $x=0.02$, indicating that the information from these weights is ignored for high dissipation, as shown in Figure 6(b). However, Figure 6(c) shows that the small weights 


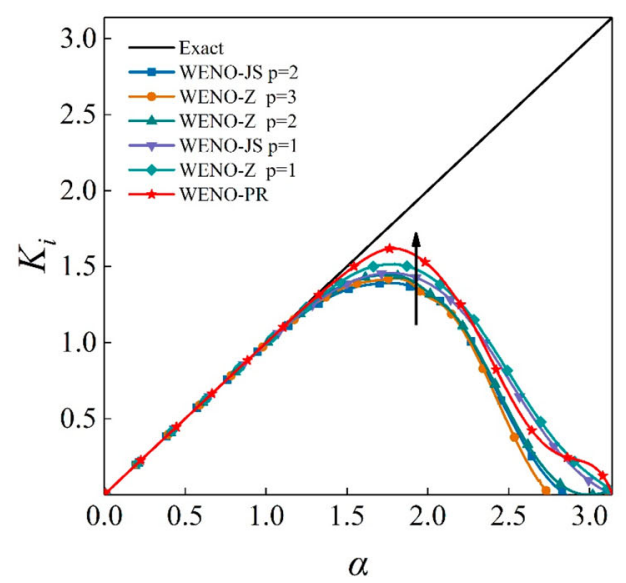

(a)

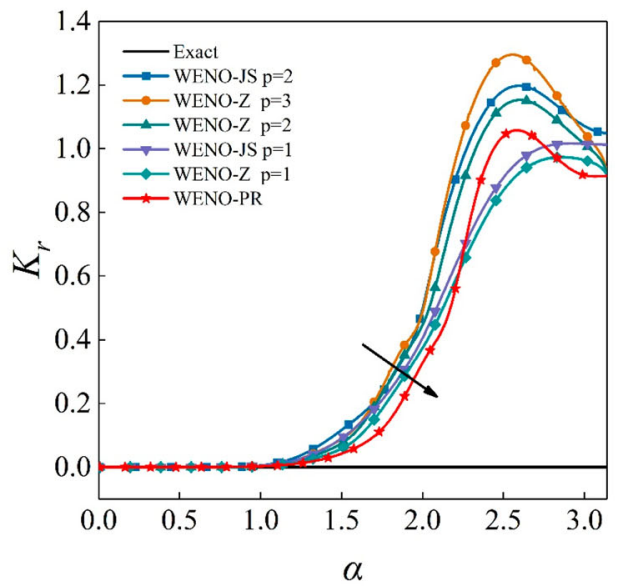

(b)

Figure 5. Numerical characteristics for the WENO-JS, WENO-Z and WENO-PR schemes. (a) Dispersion. (b) Dissipation.

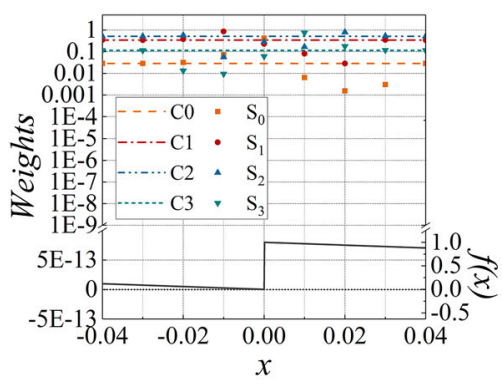

(a)

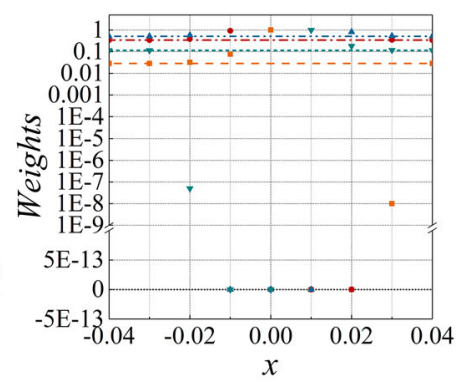

(b)

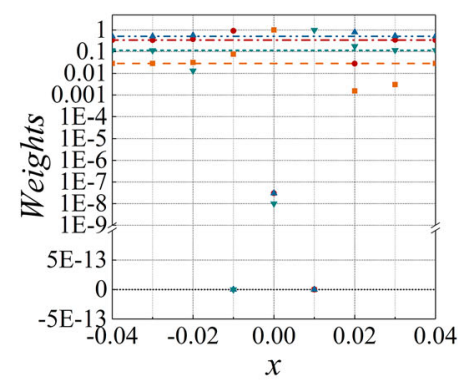

(c)

Figure 6. Distribution of ideal weights $C_{k}$ and nonlinear weights $\omega_{k}^{Z}, k=0,1,2,3$. (a) WENO-PR with $Z_{p r}=0.3 /(2 \pi)$ and $p=0.5$. The discontinuous function is shown in the lower part. (b) WENO-PR with $Z_{p r}=0.3 /(2 \pi)$ and $p=3$. (c) WENO-PR with adaptive $p$.

of the WENO-PR are concentrated near the discontinuity and a few non-zero weights which hold discontinuity information are at $x=0$. It indicates that the discontinuity is more accurately identified with an adaptive $p$. Thus, higher linear property and lower dissipation can be expected of the WENO-PR scheme.

\subsection{Accuracy test and CPU costs of the schemes}

The function $u=\cos (x), x \in[-\pi, \pi]$, with an exact derivative $\partial u / \partial x=-\sin (x)$, is chosen to test the $L_{1}$ and $L_{\infty}$ errors of the WENO-JS, WENO-Z, and WENO-PR schemes. As shown in Table 1, WENOJS reduces one order in $L_{1}$ and two orders in $L_{\infty}$ at the extreme points (Henrick, Aslam, and Powers 2005; Rathan and Raju 2018), whereas WENO-Z has the highest order of accuracy at the same locations. It is also shown that the WENO-PR maintains its actual accuracy approaching the seventh-order.
An average value of the one-time step is computed to evaluate the efficiency of the WENO-JS, WENO$\mathrm{Z}$, and WENO-PR schemes. As shown in Table 1, the computing times of the WENO-PR are $9-20 \%$ more than that of WENO-Z, whereas the CPU costs of the WENO-Z are $2-16 \%$ more than that of WENO-JS. Considering the higher resolution of the WENO-PR, the small increase in the costs is acceptable.

\section{Numerical experiments}

Several one- and two-dimensional numerical experiments are performed to assess the performance of the new scheme. The experiments solve Euler equations and adopt Steger-Warming flux vector splitting and local characteristic decomposition. Time marching is achieved via the third-order Total Variation Diminishing Runge-Kutta scheme (Suresh and Huynh 1997) which is also applicable to the seventh-order scheme 
Table 1. The $L_{1}, L_{\infty}$ errors and the rates of the convergence of the WENO-JS $\left(p=2, \varepsilon=10^{-7}\right)$, WENO-Z $\left(p=2, \varepsilon=10^{-16}\right)$ and WENO$\operatorname{PR}\left(\varepsilon=10^{-16}\right)$ schemes with the function $u=\cos (x)$.

\begin{tabular}{|c|c|c|c|c|c|c|}
\hline Methods & $N$ & $L_{1}$ error & $L_{1}$ order & $L_{\infty}$ error & $L_{\infty}$ order & CPU time \\
\hline \multirow[t]{5}{*}{ WENO-JS } & 10 & $1.60 \mathrm{E}-03$ & - & $2.88 \mathrm{E}-03$ & - & $5.10 \mathrm{E}-05$ \\
\hline & 20 & $2.80 E-05$ & 5.84 & $1.19 \mathrm{E}-04$ & 4.59 & $5.22 \mathrm{E}-05$ \\
\hline & 40 & $5.06 \mathrm{E}-07$ & 5.81 & $3.91 \mathrm{E}-06$ & 4.76 & $5.32 \mathrm{E}-05$ \\
\hline & 80 & $8.21 \mathrm{E}-09$ & 5.85 & $1.23 \mathrm{E}-07$ & 4.85 & $5.60 E-05$ \\
\hline & 160 & $1.29 \mathrm{E}-10$ & 5.88 & $3.84 \mathrm{E}-09$ & 4.89 & $6.79 E-05$ \\
\hline \multirow[t]{5}{*}{ WENO-Z } & 10 & $8.05 E-05$ & - & $1.46 \mathrm{E}-04$ & - & $5.20 E-05$ \\
\hline & 20 & $6.73 E-07$ & 6.90 & $1.18 \mathrm{E}-06$ & 6.95 & $5.34 \mathrm{E}-05$ \\
\hline & 40 & $5.34 \mathrm{E}-09$ & 6.94 & $8.63 \mathrm{E}-09$ & 7.02 & $5.48 \mathrm{E}-05$ \\
\hline & 80 & $4.19 E-11$ & 6.96 & $6.62 E-11$ & 7.03 & $6.51 \mathrm{E}-05$ \\
\hline & 160 & $3.28 \mathrm{E}-13$ & 6.97 & $5.22 \mathrm{E}-13$ & 7.02 & $7.51 \mathrm{E}-05$ \\
\hline \multirow[t]{5}{*}{ WENO-PR } & 10 & $7.88 \mathrm{E}-05$ & - & $1.24 \mathrm{E}-04$ & - & $6.20 \mathrm{E}-05$ \\
\hline & 20 & $6.74 \mathrm{E}-07$ & 6.87 & $1.45 \mathrm{E}-06$ & 6.42 & $6.30 \mathrm{E}-05$ \\
\hline & 40 & $6.57 \mathrm{E}-09$ & 6.77 & $1.83 \mathrm{E}-08$ & 6.36 & $6.51 \mathrm{E}-05$ \\
\hline & 80 & $5.80 E-11$ & 6.78 & $3.23 \mathrm{E}-10$ & 6.20 & $7.10 E-05$ \\
\hline & 160 & $4.94 \mathrm{E}-13$ & 6.80 & $5.92 \mathrm{E}-12$ & 6.16 & $9.01 \mathrm{E}-05$ \\
\hline
\end{tabular}

(Zhu et al. 2017).

$$
\begin{aligned}
& u^{(1)}=u^{n}+\Delta t \cdot L\left(u^{n}\right) \\
& u^{(2)}=\frac{3}{4} u^{n}+\frac{1}{4}\left[u^{1}+\Delta t \cdot L\left(u^{(1)}\right)\right] \\
& u^{(3)}=\frac{1}{3} u^{n}+\frac{2}{3}\left[u^{2}+\Delta t \cdot L\left(u^{(2)}\right)\right]
\end{aligned}
$$

The time step of the one-dimensional case is calculated using:

$$
\Delta t=C F L \cdot \frac{h}{\max _{j}\left(\left|u_{j}\right|+a_{j}\right)}
$$

and that of the two-dimensional case is:

$$
\Delta t=C F L \cdot \frac{\Delta t_{x} \Delta t_{y}}{\Delta t_{x}+\Delta t_{y}}
$$

where $\Delta t_{x}=\frac{h}{\max _{j, k}\left(|u|_{j, k}+a_{j, k}\right)}$, and $C F L=0.2$. The coefficient $\epsilon$ is set as a small constant as previous studies (Henrick, Aslam, and Powers 2005; Don and Borges 2013). It is noted that some computations of the WENO-Z with $p=1$ fail under the same conditions because of its too low dissipation, so only the WENOJS $\left(p=2, \varepsilon=10^{-7}\right)$, WENO-Z $\left(p=2, \varepsilon=10^{-16}\right)$ and WENO-PR $\left(\varepsilon=10^{-16}\right)$ schemes are adopted for further comparison.

\subsection{Shu-Osher and Titarev-Toro problems}

For the Shu-Osher problem, a one-dimensional Mach 3 shock-entropy wave interaction is mainly used to assess the resolution on the small and the strong waves (Shu and Osher 1988). It is specified by the following initial conditions:

$$
\begin{aligned}
& (\rho, u, p) \\
& = \begin{cases}(3.857143,2.629369,10.333333), & 0 \leq x \leq 1 \\
(1+0.2 \sin (5 x), 0,1), & 1 \leq x \leq 10\end{cases}
\end{aligned}
$$

The uniform grid points are $N=400$, and the end time is $t=1.8$. The 'exact' solution is considered as the numerical solution of the WENO-JS with $N=4000$, whereas the analytic solution is non-existent.

The second case is based on the Shu-Osher problem, in which the higher frequency entropy wave occurs (Titarev and Toro 2004). The initial condition is:

$$
\begin{aligned}
& (\rho, u, p) \\
& =\left\{\begin{array}{l}
(1.515695,0.523346,1.805),-5 \leq x \leq-4.5 \\
(1+0.1 \sin (20 \pi x), 0,1),-4.5 \leq x \leq 5
\end{array}\right.
\end{aligned}
$$

The density variation is shown with grid points $N=800$ and final time $t=5$, and the exact solution is calculated by the WENO-JS scheme with $N=8000$.

As shown in Figure 7(a), it is observed that the WENO-JS is the most dissipative one and the WENO$\mathrm{Z}$ demonstrates superior resolution than the WENOJS. The solution from the WENO-PR achieves the wave peaks, especially works well in reproducing the accurate flow features on the strong discontinuities at $x \approx 7$ where the density changes sharply after the shock waves.

As shown in Figure 7(b), the high-frequency waves of all the schemes decay after passing the shock wave, 


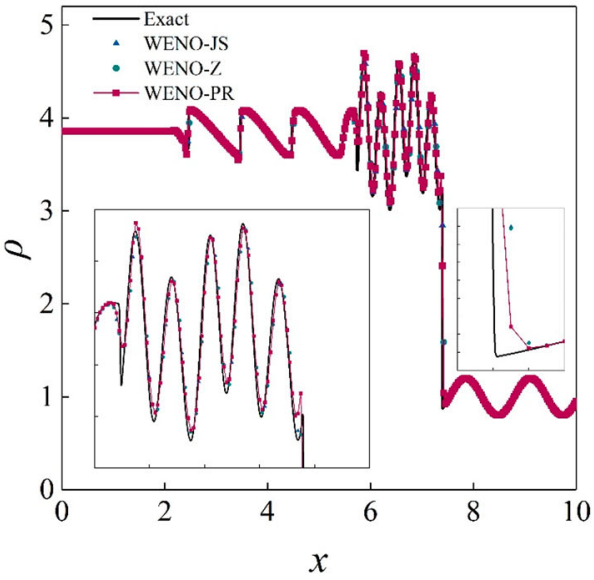

(a)

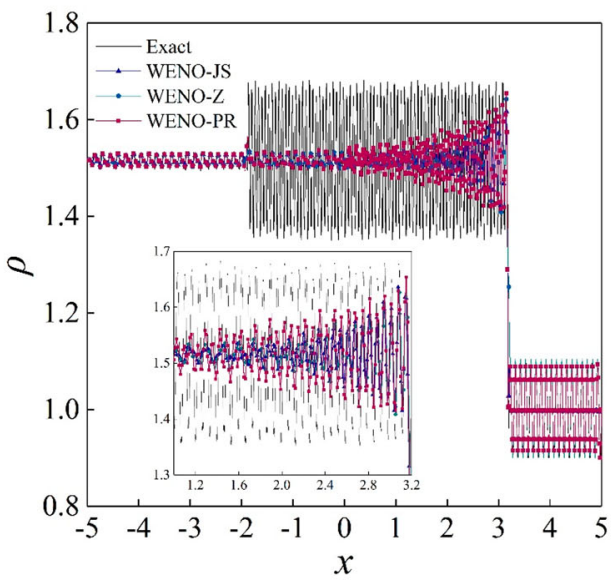

(b)

Figure 7. Density of two shock-entropy interaction problems. (a) Shu-Osher problem, $t=1.8, N=400$. (b) Titarev-Toro problem, $t=5, N=800$.

but the solution of the WENO-PR is sharper than the WENO-Z at most peaks. The WENO-PR is more sensitive to higher frequency waves. In the region with the rapidly changing waves, the WENO-PR shows better discontinuity-capturing capability. These results are consistent with the numerical characteristics as shown in Figure 5.

\subsection{Two-blast-wave problem}

This case involves complex interactions of strong shock (Hui, Li, and Li 1984; Hu, Adams, and Shu 2013; Sumi and Kurotaki 2015). The initial condition is:

$$
(\rho, u, p)=\left\{\begin{array}{l}
(1,0,1000), 0 \leq x \leq 0.1 \\
(1,0,0.01), 0.1 \leq x \leq 0.9 \\
(1,0,100), 0.9 \leq x \leq 1
\end{array}\right.
$$

and the reflective conditions are imposed on both $x=$ 0 and $x=1$. The computation is advanced till $t=$ 0.038 with $N=400$. The exact solution is calculated by the seventh-order WENO-JS scheme with $N=4000$.

The density distribution is plotted in Figure 8 which shows that the improved result is generally given by the WENO-PR, especially in the turn region at $x=$ $0.65,0.75,0.8$. It indicates that the WENO-PR stabilises for strong discontinuities.

\subsection{Double-Mach shock reflection problem}

The WENO-PR is applied to the two-dimensional double-Mach shock reflection problem (Woodward and Colella 2015). It shows that a right-moving Mach 10 shock starts up on $x=1 / 6, y=0$, and forms a $60^{\circ}$ angle between the $\mathrm{x}$-axis and itself. The parameters of the undisturbed flow before shock wave are $\rho=1.4, p=1, \gamma=1.4$. The exact post-shock conditions are used on the interval $0 \leq x \leq 1 / 6$ for the bottom boundary, and the reflective boundary condition is imposed for others. The values at the top boundary are imposed to describe the exact motion of the Mach 10 shock. The inflow and outflow boundary conditions are applied to the left and right, respectively. The solution is advanced in time up to $t=0.2$ with incremental grid points $160 \times 60,512 \times 128$ and $1024 \times 256$ on the computational domain $[0,0] \times[4,1]$. As the Mach number is significantly high, the computation can easily overflow if the numerical scheme lacks stability.

From the density contours, as shown in Figure 9, it is found that there are some basic flow characteristics around the incident shock, two Mach stems and two slip lines. For different resolutions, better rolledup small vortices and more complex shear surfaces are captured by the WENO-PR along the incline Mach stems as well as the round mushroom shape vortical structure under the incline slip line. The results from WENO-PR are better predictions of the real solution, while the WENO-JS and WENO- Z yield damped solutions owing to their larger dissipation. This test can be run using WENO-PR, but the computation overflows (occurs with negative pressure) when the WENO-Z with $p=1$ is used, which indicates that the WENO-PR is robust enough. 


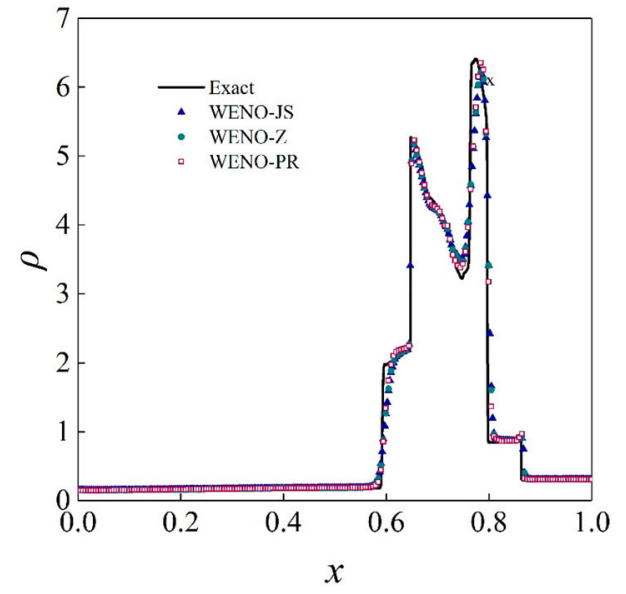

(a)

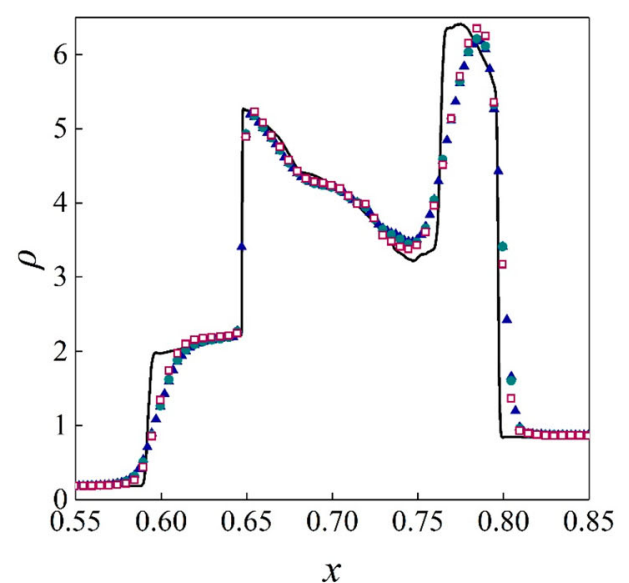

(b)

Figure 8. Density profiles in the two-blast-wave problem, $t=0.038, N=400$.


Figure 9. Density information of Double-Mach shock reflection problem plotted by 30 isolines from $\rho=1.5$ to $\rho=22.9705$, with incremental grid points. 


\subsection{Rayleigh-Taylor instability problem}

The R-T instability occurs on the unstable interface between different densities of two fluids. If heavier fluid flows into the lighter one, a number of the resulting fine flow structures will occur. Thus, this problem is usually used to test the resolution of a numerical scheme (Shi, Zhang, and Shu 2003). The twodimensional Euler equation in conservative form is:

$$
\frac{\partial U}{\partial t}+\frac{\partial F_{1}(U)}{\partial x}+\frac{\partial F_{2}(U)}{\partial y}=S
$$

where source item $S=(0, \rho, 0, \rho v)^{T}$. The initial condition of the above equation is:

$$
\begin{aligned}
& (\rho, u, v, p) \\
& = \begin{cases}(2,0,-0.025 c \cdot \cos (8 \pi x), 2 y+1), & 0 \leq y \leq 0.5 \\
(1,0,-0.025 c \cdot \cos (8 \pi x), 2 y+1.5), & 0.5 \leq y \leq 1\end{cases}
\end{aligned}
$$

where $c$ is the speed of sound expressed as $c=\sqrt{(\gamma p / \rho)}, \gamma=5 / 3$. The computational domain is $[0,0.25] \times[0,1]$. The $120 \times 480$ uniform grids are taken and the simulation till $t=1.95$ is performed.

The resulting density contours are shown in Figure 10. It can be seen that heavier fluid flows into the other along the vertical axis, forming mushroom pattern structures. There are two large vortices under the mushroom head and several small vortices rolling up beside the central flow column.

Although all the WENO schemes distinguish the basic structures, the isolines from the WENO- PR are more intensive inside the mushroom head capturing small vortices. Some spurious oscillations are removed at $(0.12,0.75)$ compared to the WENO-Z. In the remaining smooth regions, the WENO-PR and WENO-Z schemes agree well, indicating a reliable solution and better resolution results given by the WENO-PR. This case shows that the WENO-PR has a better balance in capturing small-scale structures and avoiding numerical oscillations.

\subsection{Two-dimensional implosion case}

This example is an unsteady flow in a two-dimensional container (Hui, Li, and Li 1999). The initial condition is:

$$
(\rho, u, v, p)= \begin{cases}(1,0,0,1), & x^{2}+y^{2} \leq 0.15^{2} \\ (0.125,0,0,0.14), & \text { else }\end{cases}
$$

At $t=0$, the flow field is separated into two parts, the inner one of which is a circle. If the computation starts, the circular region begins to interact with the outer region. Owing to the reflective boundary condition applied except the outflow condition on the upper boundary, the flow becomes more complex. As shown in Figure 11, complex flow details are identified by the WENO-Z and WENO-PR, and the latter is better. It can be seen that the WENO$\mathrm{PR}$ achieves a compromise between resolution and robustness.

\subsection{Shock-Bubble interaction problem}

This case describes a normal shock wave propagates through air from left to right and impacts on a cylindrical helium bubble (Bagabir and Drikakis 2001). It is a challenging problem to determine the resolution and the robustness of the numerical schemes. The problem is simplified by assuming air and helium as perfect gas with the ratio of specific heat capacities $\gamma=1.4$. The initial conditions are given by:

$$
(\rho, u, v, p)= \begin{cases}(1,-6,0,1) & \text { pre shocked air } \\ (5.799,5.5750,0,167.833), & \text { post shocked air } \\ (0.138,-6,0,1), & \text { helium bubble }\end{cases}
$$

Initially, the radius of the helium bubble is 0.15 with the centre at $(0.25,0)$ and a shock wave moves from $(0.05,0)$. Notably, $u=-6$ is applied to maintain the bubble in the computation domain. The inflow condition is applied with post-shock condition to the left boundary and the outflow condition is considered to the right boundary. The reflective conditions are given for the upper and lower boundaries.

Figure 12 provides a comparison of the three schemes at $t=0.075$. The strong shock smashes into the bubble and generates a group of vortices, shown by the distorted isolines. As shown in Figure 12(d), while the relatively sketchy solutions are given by the WENO-JS and WENO-Z, richer structures are captured by the WENO-PR, for example, the result contains greater distortion near $(0.6,0.25)$, indicating that linearity is provided by the adaptive $p$. Also, the WENO-Z with $p=1$ overflows in this test owing to its instability. It indicates that the resolution and robustness of the WENO-PR are improved. 


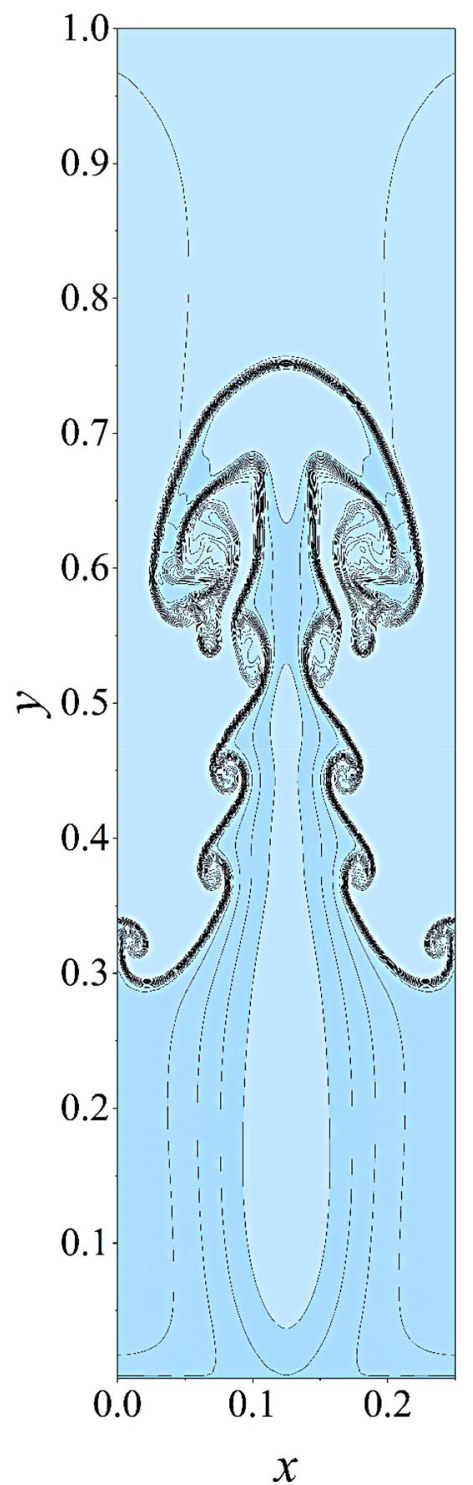

(a)

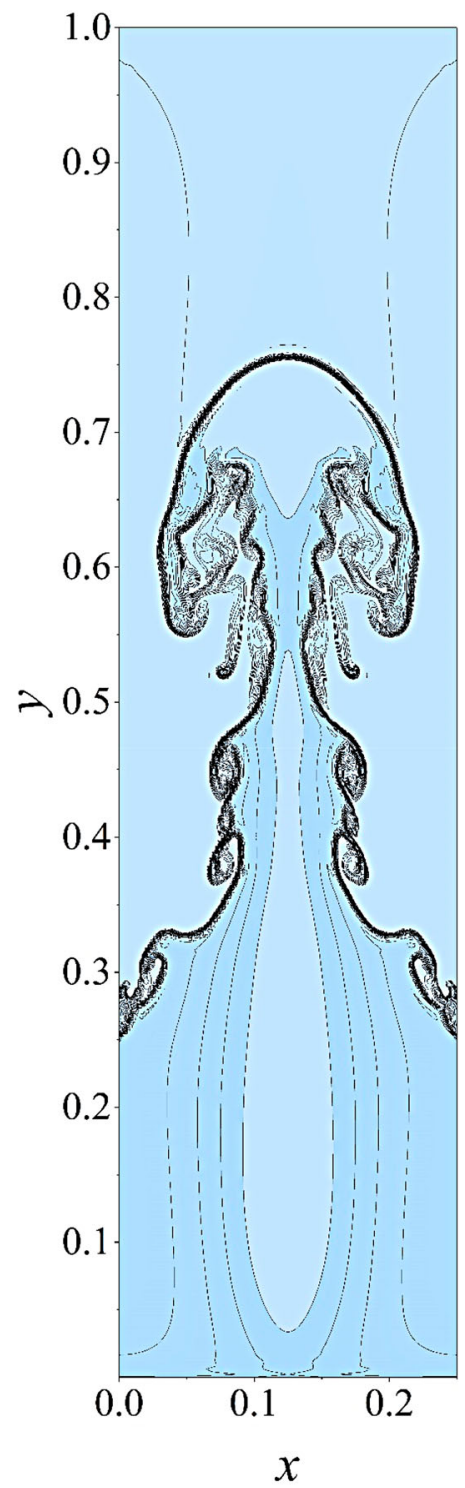

(b)



(c)

Figure 10. Density of R-T instability problem plotted by 15 isolines from $\rho=0.9952269$ to 2.14589 with $N=120 \times 480$. (a) WENO-JS. (b) WENO-Z. (c) WENO-PR.

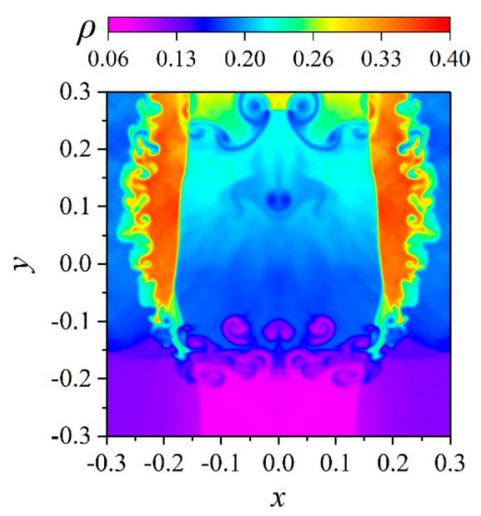

(a)

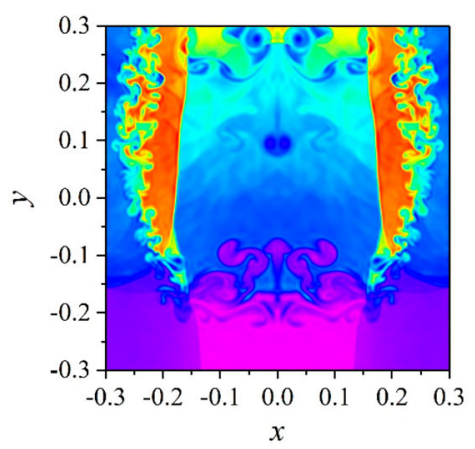

(b)

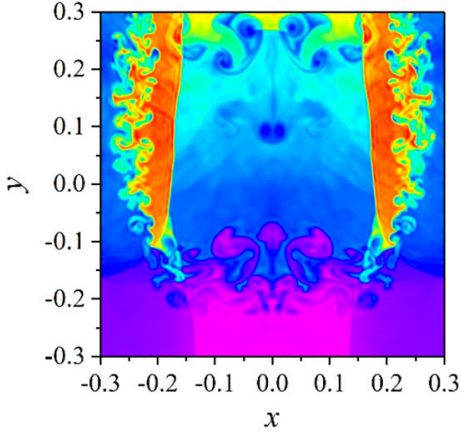

(c)

Figure 11. Density of implosion problem with $N=256 \times 256$. (a) WENO-JS. (b) WENO-Z. (c) WENO-PR. 


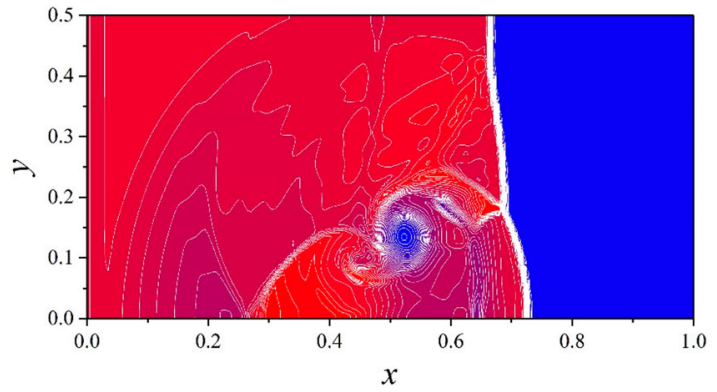

(a)

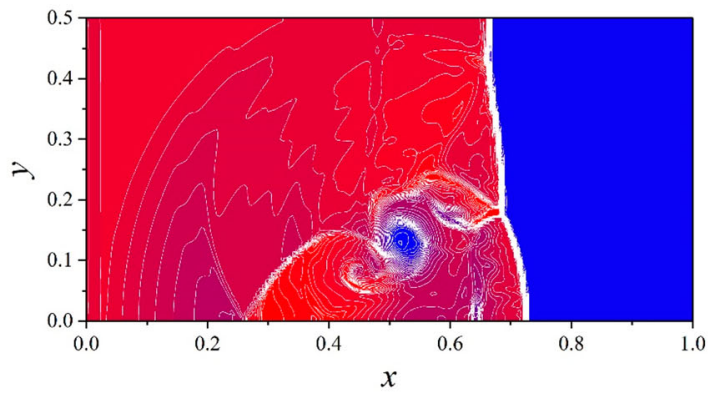

(c)

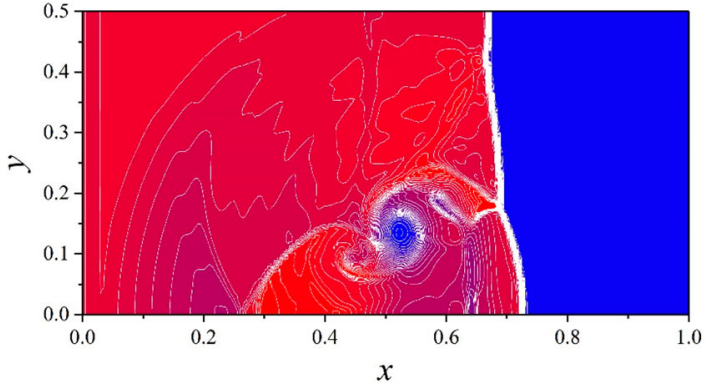

(b)

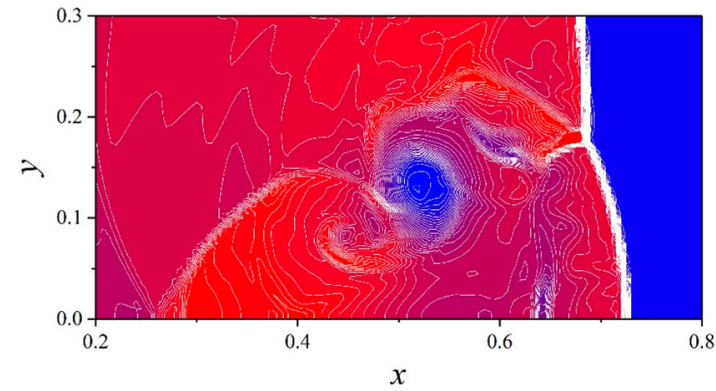

(d)

Figure 12. Density of Shock-Bubble interaction problem plotted by 33 isolines from $\rho=0.6$ to 7.1 , with $N=200 \times 100$. (a) WENO-JS. (b) WENO-Z. (c) WENO-PR. (d) Details for WENO-PR.

\section{Conclusions}

In this paper, a new WENO scheme (WENO-PR) is proposed based on the seventh-order WENO-Z scheme. In the WENO-PR, the high-order smoothness indicator $\tau_{7}$ is modified by a limiting function and a parameter $Z_{p r}$, which leads to larger weights assigned at the discontinuities. Also, an expression of the real-number adaptive $p$ controlled by a wavenumber identifier is proposed. Owing to the adaptive $p$, the numerical dissipation can be adjusted reasonably in low- and high-wavenumber regions, resulting in less dissipation and more robustness. Furthermore, the nonlinear spectral analysis is adopted to determine the expressions of $\tau_{7}$ and $p$. The weights analysis proves that the numerical characteristics of the WENO-PR are better than the WENOJS and WENO-Z schemes. Canonical test problems are applied to test the new scheme, including onedimensional Shu-Osher, Titarev-Toro and two-blastwave problems, two-dimensional Double-Mach shock reflection, Rayleigh-Taylor instability, implosion and Shock-Bubble interaction problems. The tests illustrate that the WENO-PR is less dissipative and more robust with little increase in CPU cost. The methods for improving $\tau_{7}$ and $p$ in this paper provide different ways to control dissipation and assign the weights, and they may be further promoted by more accurate wavenumber identifier and modification.

\section{Acknowledgments}

The authors thank National Supercomputer Center in Tianjin (NSCC-TJ), and National Supercomputer Center in Guang Zhou (NSCC-GZ) for providing computer time.

\section{Disclosure statement}

No potential conflict of interest was reported by the author(s).

\section{Funding}

This work was supported by the National Key Research and Development Program of China (2019YFA0405300, 2016YFA0401200) and NSFC Projects (91852203), Science Challenge Project (TZ2016001), and Strategic Priority Research Program of Chinese Academy of Sciences (Grant Nos. XDC01000000).

\section{ORCID}

Xin-Liang Li (1) http://orcid.org/0000-0002-4264-9620

\section{References}

Aràndiga, F., M. C. Martí, and P. Mulet. 2014. "Weights Design for Maximal Order WENO Schemes." Journal of Scientific Computing 60 (3): 641-659. 
Arshed, G. M., and O. U. Khan. 2019. "Problem-independent Nonlinear Switch for Newly Designed WENO-BO-Z Scheme." International Journal of Computational Fluid Dynamics 33 (1-2): 59-76.

Bagabir, A., and D. Drikakis. 2001. "Mach Number Effects on Shock-Bubble Interaction." Shock Waves 11 (3): 209-218.

Balsara, D. S., and C. W. Shu. 2000. "Monotonicity Preserving Weighted Essentially Non-oscillatory Schemes with Increasingly High Order of Accuracy." Journal of Computational Physics 160 (2): 405-452.

Borges, R., M. Carmona, B. Costa, and W. S. Don. 2008. “An Improved Weighted Essentially Non-oscillatory Scheme for Hyperbolic Conservation Laws." Journal of Computational Physics 227 (6): 3191-3211.

Castro, M., B. Costa, and W. S. Don. 2011. "High Order Weighted Essentially Non-oscillatory WENO-Z Schemes for Hyperbolic Conservation Laws." Journal of Computational Physics 230 (5): 1766-1792.

Don, W. S., and R. Borges. 2013. "Accuracy of the Weighted Essentially Non-oscillatory Conservative Finite Difference Schemes." Journal of Computational Physics 250: 347-372.

Fan, P., Y. Shen, B. Tian, and C. Yang. 2014. "A new Smoothness Indicator for Improving the Weighted Essentially Nonoscillatory Scheme." Journal of Computational Physics 269 (1): 329-354.

Fu, L., X. Y. Hu, and N. A. Adams. 2016. "A Family of Highorder Targeted ENO Schemes for Compressible-fluid Simulations." Journal of Computational Physics 305: 333-359.

Fu, L., X. Y. Hu, and N. A. Adams. 2017. “Targeted ENO Schemes with Tailored Resolution Property for Hyperbolic Conservation Laws." Journal of Computational Physics 349: 97-121.

Fu, L., X. Y. Hu, and N. A. Adams. 2018. "A New Class of Adaptive High-order Targeted ENO Schemes for Hyperbolic Conservation Laws." Journal of Computational Physics 374: 724-751.

Gande, N. R., Y. Rathod, and S. Rathan. 2017. "Third-order WENO Scheme with a New Smoothness Indicator." International Journal for Numerical Methods in Fluids 85 (2): 90-112.

Ha, Y., C. H. Kim, Y. J. Lee, and J. Yoon. 2013. “An Improved Weighted Essentially Non-oscillatory Scheme with a New Smoothness Indicator." Journal of Computational Physics 232 (1): 68-86.

Harten, A. 1983. "High Resolution Schemes for Hyperbolic Conservation Laws." Journal of Computational Physics 49 (3): 357-393.

Harten, A. 1989. "ENO Schemes with Subcell Resolution." Journal of Computational Physics 83 (1): 148-184.

Harten, A., B. Engquist, S. Osher, and S. R. Chakravarthy. 1987. "Uniformly High Order Accurate Essentially Non-oscillatory Schemes, III." Journal of Computational Physics 71 (2): 231-303.

Harten, A., S. Osher, B. Engquist, and S. R. Chakravarthy. 1986. "Some Results on Uniformly High Order Accurate Essentially Non-oscillatory Schemes." Applied Numerical Mathematics 2 (3-5): 347-377.
Henrick, A. K., T. D. Aslam, and J. M. Powers. 2005. “Mapped Weighted Essentially Non-oscillatory Schemes: Achieving Optimal Order Near Critical Points." Journal of Computational Physics 207 (2): 542-567.

Hu, X. Y., N. A. Adams, and C. W. Shu. 2013. "Positivitypreserving Method for High-order Conservative Schemes Solving Compressible Euler Equations." Journal of Computational Physics 242 (1): 169-180.

Hu, X. Y., Q. Wang, and N. A. Adams. 2010. “An Adaptive Central-upwind Weighted Essentially Non-oscillatory Scheme." Journal of Computational Physics 229 (23): 89528965.

Hui, W. H., P. Y. Li, and Z. W. Li. 1984. "The Numerical Simulation of Two-dimensional Fluid Flow with Strong Shocks." Journal of Computational Physics 153 (2): 596-637.

Hui, W. H., P. Y. Li, and Z. W. Li. 1999. "A Unified Coordinate System for Solving the Two-dimensional Euler Equations.” Journal of Computational Physics 153 (2): 596-637.

Jiang, G. S., and C. W. Shu. 1996. "Efficient Implementation of Weighted ENO Schemes." Journal of Computational Physics 126 (1): 202-228.

Kim, C. H., Y. Ha, and J. Yoon. 2016. "Modified Nonlinear Weights for Fifth-order Weighted Essentially Nonoscillatory Schemes." Journal of Scientific Computing 67 (1): 299-323.

Kitamura, K., and T. Nonomura. 2017. "Assessment of WENOExtended Two-fluid Modelling in Compressible Multiphase Flows." International Journal of Computational Fluid Dynamics 31 (3): 188-194.

Li, X. G., G. D. Li, and Y. B. Ge. 2019. "Improvement of Thirdorder Finite Difference WENO Scheme at Critical Points." International Journal of Computational Fluid Dynamics 34: $1-13$.

Li, Y. S., C. Yan, J. Yu, and H. K. Liu. 2017. "A New Highaccuracy Scheme for Compressible Turbulent Flows." International Journal of Computational Fluid Dynamics 31 (9): 362-378.

Li, L., C. P. Yu, Z. Chen, and X. L. Li. 2016. "Resolutionoptimised Nonlinear Scheme for Secondary Derivatives." International Journal of Computational Fluid Dynamics 30 (2): 107-119.

Liu, X. D., S. Osher, and T. Chan. 1994. "Weighted Essentially Non-oscillatory Schemes." Journal of Computational Physics 115 (1): 200-212.

Liu, X. L., S. H. Zhang, H. X. Zhang, and C. W. Shu. 2015. "A New Class of Central Compact Schemes with Spectrallike Resolution II." Journal of Computational Physics 284 (C): 133-154.

Martín, M. P., E. M. Taylor, M. Wu, and V. G. Weirs. 2006. "A Bandwidth-optimized WENO Scheme for the Effective Direct Numerical Simulation of Compressible Turbulence." Journal of Computational Physics 220 (1): 270-289.

Pirozzoli, S. 2002. "Conservative Hybrid Compact-WENO Schemes for Shock-turbulence Interaction." Journal of Computational Physics 178 (1): 81-117.

Rathan, S., and G. N. Raju. 2018. "Improved Weighted ENO Scheme Based on Parameters Involved in Nonlinear 
Weights." Applied Mathematics and Computation 331: 120-129.

Shi, J., Y. T. Zhang, and C. W. Shu. 2003. "Resolution of High Order WENO Schemes for Complicated Flow Structures." Journal of Computational Physics 186 (2): 690696.

Shu, C. W. 1987. "TVB Uniformly High-order Schemes for Conservation Laws." Mathematics of Computation 49 (179): 105-121.

Shu, C. W., and S. Osher. 1988. "Efficient Implementation of Essentially non-Oscillatory Shock-capturing Schemes." Journal of Computational Physics 77 (2): 439-471.

Shu, C. W., and S. Osher. 1989. "Efficient Implementation of Essentially Non-oscillatory Shock-capturing Schemes, II.” Journal of Computational Physics 83 (1): 32-78.

Sumi, T., and T. Kurotaki. 2015. "A New Central Compact Finite Difference Formula for Improving Robustness in Weighted Compact Nonlinear Schemes." Computers and Fluids 123: 162-182.

Suresh, A., and H. T. Huynh. 1997. "Accurate Monotonicitypreserving Schemes with Runge-Kutta Time Stepping.” Journal of Computational Physics 136 (1): 83-99.

Titarev, V. A., and E. F. Toro. 2004. "Finite-volume WENO Schemes for Three-dimensional Conservation Laws." Journal of Computational Physics 201 (1): 238-260.
Tong, F. L., X. L. Li, Y. H. Duan, and C. P. Yu. 2017. “Direct Numerical Simulation of Supersonic Turbulent Boundary Layer Subjected to a Curved Compression Ramp." Physics of Fluids 29 (12): 125101.

Wang, Y. H., B. S. Wang, and W. S. Don. 2019. “Generalized Sensitivity Parameter Free Fifth Order WENO Finite Difference Scheme with z-type Weights." Journal of Scientific Computing 78: 1-30.

Wang, L., and S. Wu. 2015. "Hybrid Finite Difference Schemes Without Free Parameters." Journal of Beijing University of Aeronautics and Astronautics 41 (2): 318-322.

Woodward, P., and P. Colella. 2015. "The Numerical Simulation of Two-dimensional Fluid Flow with Strong Shocks." Journal of Computational Physics 54 (1): 115-173.

Wu, M., and M. P. Martín. 2007. “Direct Numerical Simulation of Supersonic Turbulent Boundary Layer Over a Compression Ramp." AIAA Journal 45 (4): 879-889.

Zhou, Q., F. He, and M. Y. Shen. 2012. "A Family of Efficient High-order Hybrid Finite Difference Schemes Based on WENO Schemes." International Journal of Computational Fluid Dynamics 26 (4): 205-229.

Zhu, X. K., C. P. Yu, F. L. Tong, and X. L. Li. 2017. "Numerical Study on Wall Temperature Effects on Shock Wave/Turbulent Boundary-Layer Interaction." AIAA Journal 55 (1): 131-140. 